\title{
Powering the planet with solar fuel
}

\author{
With energy swiftly rising to the top of the world's agenda, Harry B. Gray at the California Institute of Technology \\ looks at how chemistry can help to harness the power of the Sun to meet the world's energy needs.
}

O ne of the grand challenges of twenty-first century chemistry is to convert abundant energy-poor molecules to energy-rich molecules using sunlight as the energy source. We need to replace fossil fuels with solar fuels such as hydrogen from water, or methanol from water and carbon dioxide. Solar fuels could be used round the clock, as the chemical energy stored during the day can be converted to mechanical or electrical power both day and night.

Viable renewable energy systems will require chemical catalysts that are far superior to any we have now. We must have catalysts made from dirt-cheap, Earth-abundant materials. When we say dirt-cheap, we mean that quite literally. Platinum is a wonderful and versatile catalyst, but there isn't enough of it on the planet to meet our energy needs (and we must save some for jewellery and best-selling albums!). Our catalysts must not only be cheap, but they must also last for years. That is a very tall order.

Long ago, nature figured out how to use abundant metallic elements in combination with proteins to activate small molecules. However, the organic frameworks of these biological molecules are readily degraded by oxidative and other chemical processes, greatly limiting their lifetimes. Nature copes with limited lifetimes - a living system can rebuild the key components it needs - but we must find other ways to deal with the problem. We and many other investigators have attempted to construct functional mimics of enzyme active sites, but one lesson we have learnt after much hard work is that these special metallocentres perform well only when they are embedded in their folded protein cages $^{1}$. The good news is that there is much activity in the field at present; indeed, we have every reason to believe that biological inorganic chemists will soon be able to design and construct bioinspired materials that will efficiently catalyse water oxidation and reduction.

What about pure inorganic materials? Rust and fool's gold could be an ideal catalyst pair were it not for lattice defects that cause photoinduced electrons and holes to recombine before they can be used productively. But any system that might be workable on the scale we need will probably use a robust metal

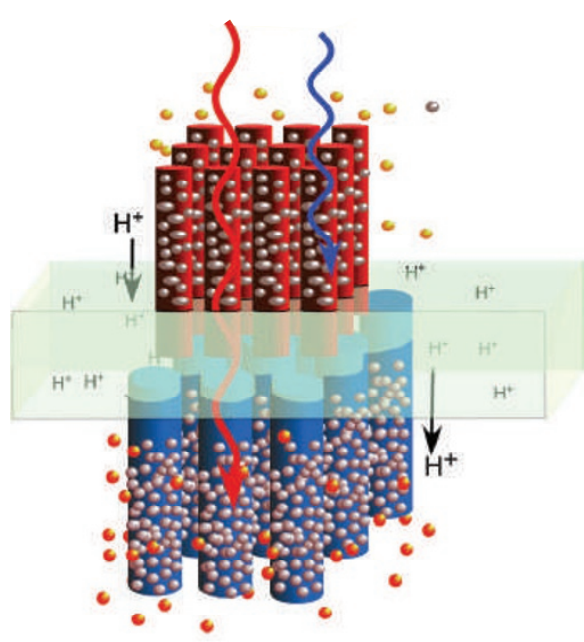

A solar fuel cell. The blue portion of sunlight is absorbed by the metal oxide anode (red), where it oxidizes water to release protons. The red portion passes through to be absorbed by the silicon nanorod cathode (blue), which combines these protons with electrons to make hydrogen for fuel. The membrane (light blue) must be permeable to the generated protons and conduct electrons between the two electrodes. The spheres in the region of the anode represent oxygen molecules; spheres in the region of the cathode represent hydrogen molecules. The hydrogen to oxygen ratio is 2:1.

oxide - literally a rock - to oxidize water to oxygen ${ }^{2}$; and any of a number of closely related materials, including metal sulfides ${ }^{3}$, to reduce water to hydrogen.

Our Solar Fuel Center for Chemical Innovation (CCISolar) ${ }^{4}$ involves many experimentalists and theorists in an interdisciplinary effort with the goal of building an assembly of nanorod catalysts to split water. The device that we envisage features nanorod anodes and cathodes of dissimilar materials embedded in a membrane to harvest sunlight (pictured). Blue light is absorbed on the anode side of the membrane, interacting with a catalyst to oxidize water and release protons. Red light is absorbed on the cathode side, where protons combine with electrons to make hydrogen. CCISolar researchers have made good progress in several areas, especially on the development of nanorod array photoelectrodes ${ }^{5}$, catalysts for water oxidation $^{6}$ and reduction ${ }^{7}$, and bioinspired photosystems ${ }^{8}$. However, we and other investigators worldwide have a long way to go before an efficient water splitter made from Earthabundant materials is ready for deployment on a large scale.

We must solve many fundamental chemical problems before we can claim victory. But if we stay the course, we could have working solar-fuel plants before 2050. Facilities situated in coastal metropolitan areas could use sea water as the source of hydrogen and oxygen. The hydrogen produced from water splitting could then be run through adjoining fuelcell electric power plants where the pure water by-product could go directly into the municipal water system. Imagine meeting local energy and clean water needs at the same time!

We have an even grander vision. Some time in the future we will be able to put three components of our atmosphere carbon dioxide, nitrogen and oxygen along with sea water into solar reactors to make not only fuels, electricity and pure water, but polymers, food and almost everything else we need. We have been taking from nature since the beginning of time, consuming the oil, gas and coal given to us by thousands of millions of years of photosynthesis. This is the century in human history when we will start paying back with the capital generated through fundamental research in chemistry.

Harry B. Gray is in the Division of Chemistry and Chemical Engineering at the California Institute of Technology, Pasadena, California 91125, USA. e-mail:hbgray@caltech.edu

Acknowledgements

I would like to thank my colleagues Jay R. Winkler, Bruce S. Brunschwig and Douglas L. Smith for their contributions to this article.

\section{References}

1. Gray, H. B., Malmstrom, B. G. \& Williams, R. J. P. J. Biol. Inorg. Chem. 5, 551-559 (2000).

2. Harriman, A., Pickering, I. J., Thomas, J. M. \& Christensen, P. A. J. Chem. Soc., Faraday Trans. I 84, 2795-2806 (1988).

3. Jaramillo, T. F. et al. Science 317, 100-102 (2007)

4. http://www.ccisolar.caltech.edu

5. Spurgeon, J. M., Atwater, H. A. \& Lewis, N. S. J. Phys. Chem. C 112, 6186-6193 (2008)

6. Kanan, M. W. \& Nocera, D. G. Science 321, 1072-1075 (2008)

7. Hu, X. L., Brunschwig, B. S. \& Peters, J. C. J. Am. Chem. Soc. 129, 8988-8998 (2007).

8. Shih, C. et al. Science 320, 1760-1762 (2008) 


\section{ERRATUM}

Powering the planet with solar fuel

Harry B. Gray

Nature Chemistry 1, 7 (2009); corrected after print: 19 March 2009.

In the version of this article originally published, the ratio of hydrogen to oxygen given in the figure caption was incorrect; it should have read 2:1, and has now been corrected in the HTML and PDF versions. 